\title{
Study of Terahertz Generation in Magnetized Plasma Via Self Focused Ultra-Relativistic Laser Beam
}

\author{
Monika Singh $^{\text {)a }}$ Munther B. Hassan $^{\text {bb }}$, A. H. Al- Janabi ${ }^{\text {c }}$ \\ .R. P. Sharma ${ }^{\text {)d }}$ Narender Kumar ${ }^{\text {ee }}$ \\ ${ }^{a), d)}$ Centre for Energy Studies, Indian Institute of Technology Delhi, New Delhi-110016, India \\ ${ }^{b)}$ Department of Physics, Faculty of Science, University of Kufa, Najaf, Iraq. \\ ${ }^{c}$ Institute of Laser for Postgraduate studies, University of Baghdad, Baghdad, Iraq. \\ ${ }^{e)}$ Sri Venkateswara College, University of Delhi, New Delhi-110021, India
}

\begin{abstract}
The paper presents a theoretical model for terahertz (THz) radiation generation at the difference frequency of two waves namely x-mode laser and upper hybrid wave (UHW). By considering relativistic nonlinearity, filamentation (self focusing) of $x$-mode laser propagating perpendicular to the direction of ambient magnetic field in plasma is first investigated. This resulting localized beam interacts with the UHW to produce a nonlinear current at the THz frequency range. The differential equation for beam-width parameter is obtained by using the dielectric function within the paraxial ray approximations. Subsequently, an expression for THz electric field is derived by including the effect of relativistic self-focusing of pump laser. Our analytical and numerical calculations show that the relativistic self focusing and magnetic field play a crucial role to enhance the $\mathrm{THz}$ radiation generation.
\end{abstract}

Keywords: THz radiation; Self-focusing, relativistic nonlinearity; Plasma waves; magnetized plasma

\section{Introduction}

From the few decades, $\mathrm{THz}$ domain has witnessed significant attention and improvements and has become an important research tool in many important areas of science and engineering because of its sources got diverse applications in biological imaging, remote sensing and inspections for illegal drugs and bombs in mail [1-3].

Plasma [4], as a nonlinear medium, has the ability to generate high power coherent and incoherent $\mathrm{THz}$ radiations in comparison to other conventional $\mathrm{THz}$ generation mechanisms [5, 6].

The self-focusing phenomenon $[7,8]$ is dependent on the intensity distribution along the beam wave front. It is a topic of utmost importance and it effects a lot of fields such as optical harmonic generation $[9,10]$, inertial confinement fusion [11, 12], ionospheric modification [13,14], laser-electron acceleration [15,16] and $\mathrm{THz}$ emission obtained by the nonlinear Laser-plasma interactions [17-24]. The generation of $\mathrm{THz}$ radiations from laser filaments is also high and has been investigated extensively. The $\mathrm{THz}$ frequency waves of few hundred nJ pulse energy was experimentally observed [23] with two fold magnitude magnification in the power level of $\mathrm{THz}$ radiation which is due to the filamentation of air by the two colour femtosecond lasers. A scheme of THz wave's emission by the plasma filament created by a high power fs laser pulse has been investigated by a 2D-PIC simulation [24]. A THz wave having linear polarisation (in the plane containing the static electric field) was investigated from laser filaments in the presence of a static, transverse electric field $10 \mathrm{KV} / \mathrm{cm}$ in plasma. Filamentation is also found responsible for three fold energy enhancement of $\mathrm{THz}$ wave by Houard $e t$ al. [20] .

It has become possible to investigate the advanced regime of relativistic laser-matter interactions because of the occurrence of tetra watt lasers at centred intensities as high as $10^{18} \mathrm{~W} / \mathrm{cm}^{2}$. The self-focusing and self-channelling of an intense laser beam at relativistic power in a review comprehensively [33, 34].

Three dimensional particles in cell simulations have been used to form the filaments and generate the multimegagauss magnetic field during the laser plasma interaction in relativistic regime [35].

We study $\mathrm{THz}$ frequency radiation generation scheme, based on the non-linear interaction of $\mathrm{x}$-mode laser with UHW. For this purpose we have studied the relativistic self-focusing associated with x-mode laser in the magnetized plasma. A proper match of pump wave frequency $\left(\omega_{0}\right)$ and UHW frequency $\left(\omega_{1}\right)$ gives an electromagnetic radiation of THz frequency $\left(\omega_{2}\right)$ via the beating current. Thus, there is a resonant excitation of the $\mathrm{THz}$ radiation through the proper matching condition. 
Section 2 describes the analytical study of the self focusing of extraordinary mode of laser beam through the magnetized plasma in the relativistic domain. Section 3 represents the UHW and THz dispersion relations respectively. We have given the discussion and conclusion of results in sections 4and 5.

\section{Relativistic Self Focusing of X- Mode Laser Beam}

The extraordinary laser beam (x-mode) propagation inside a homogeneous magnetized plasma along $x$ direction and perpendicular on an external magnetic field $\vec{B}_{0}$ aligned in $z$-direction. The variation of x-mode electric field $\vec{E}_{0}$ may be written as follows

$$
\vec{E}_{0}=\left(E_{x} \hat{x}+E_{y} \hat{y}\right) \exp -i\left(\omega_{0} t-k_{0} x\right),
$$

where, $\omega_{0}$ and $k_{0}$ represents the angular frequency and propagation wave vector respectively which is related by

$$
k_{0}=\left(\varepsilon_{r}\right)^{\frac{1}{2}}\left(\frac{\omega_{0}}{c}\right)
$$

$c$ is the light speed and $\varepsilon_{r}$ is the dielectric constant in relativistic case which is given by

$$
\varepsilon_{r}=1-\frac{\frac{\omega_{p e}^{2}}{\gamma}\left(\omega_{0}^{2}-\frac{\omega_{p e}^{2}}{\gamma}\right)}{\omega_{0}^{2}\left(\omega_{0}^{2}-\frac{(\gamma-1) \omega_{p e}^{2}+\omega_{u}^{2}}{\gamma^{2}}\right)},
$$

where, $\omega_{p e}=\left(\frac{4 \pi n_{0} e^{2}}{m_{0}}\right)^{\frac{1}{2}}, \omega_{u}=\left(\omega_{p e}^{2}+\omega_{c e}^{2}\right)^{\frac{1}{2}}$ and $\omega_{c e}=\frac{e B_{0}}{m_{0} c}$ are the angular frequencies of the electron plasma wave, upper hybrid wave and electron cyclotron respectively, also $n_{0}, m_{0}$ and $-e$ represent the plasma density, electron rest mass and electron charge respectively.

The relativistic factor $\gamma$ in Eq. (3) refers to the variation of the dielectric constant due to the increase in the electron mass at relativistic case is given by [36]

$$
\gamma=\left(1+\frac{v_{0}^{2}}{2 c^{2}}\right)
$$

where $v_{0}$ is the electron oscillating velocity imparted by electric field of laser beam

$$
v_{0}=\frac{1}{\sqrt{2}}\left(v_{0 x} \cdot v_{0 x}^{*}+v_{0 y} \cdot v_{0 y}^{*}\right)^{\frac{1}{2}} \text {, }
$$

The equation for the electric field of high power laser beam is given as

$$
m_{0} \gamma \frac{\partial}{\partial t} \vec{v}_{0}=-e \vec{E}_{0}-\frac{e}{c}\left(\vec{v}_{0} \times \vec{B}_{0}\right),
$$

From Eq. (6), one may get the velocity components $v_{0 x}$ and $v_{0 y}$ of the electron as

$$
\begin{gathered}
v_{0 x}=\frac{-i e}{m_{0} \gamma \omega_{0}\left(1-\frac{\omega_{c e}^{2}}{\gamma^{2} \omega_{0}^{2}}\right)} E_{x}-\frac{e \omega_{c e}}{m_{0} \gamma^{2} \omega_{0}^{2}\left(1-\frac{\omega_{c e}^{2}}{\gamma^{2} \omega_{0}^{2}}\right)} E_{y} . \\
v_{0 y}=\frac{e \omega_{c e}}{m_{0} \gamma^{2} \omega_{0}^{2}\left(1-\frac{\omega_{c e}^{2}}{\gamma^{2} \omega_{0}^{2}}\right)} E_{x}-\frac{i e}{m_{0} \gamma \omega_{0}\left(1-\frac{\omega_{c e}^{2}}{\gamma^{2} \omega_{0}^{2}}\right)} E_{y} .
\end{gathered}
$$

For the $\mathrm{x}$-mode laser, one can introduce the following equations [37]

$\nabla \cdot \underline{\underline{\varepsilon}} \cdot \vec{E}_{0}=0$ 


$$
\begin{aligned}
& E_{x}=\frac{-\varepsilon_{x y}}{\varepsilon_{y y}} E_{y}, \\
& \varepsilon_{y y}=1-\frac{\omega_{p e}^{2}}{\gamma \omega_{0}^{2}\left(1-\frac{\omega_{c e}^{2}}{\gamma^{2} \omega_{0}^{2}}\right)}, \\
& \varepsilon_{x y}=\frac{i \omega_{p e}^{2} \omega_{c e}}{\gamma^{2} \omega_{0}^{3}\left(1-\frac{\omega_{c e}^{2}}{\gamma^{2} \omega_{0}^{2}}\right)},
\end{aligned}
$$

using Eqs.(9-12), then Eq.(7) and Eq.(8) will take the following forms

$$
v_{0 x}=\frac{-e}{m_{0} \gamma \omega_{0}\left(1-\frac{\omega_{c e}^{2}}{\gamma^{2} \omega_{0}^{2}}\right)}\left[\frac{\left(\frac{\omega_{c e}}{\gamma \omega_{0}}\right)\left(\frac{\omega_{p e}^{2}}{\gamma \omega_{0}^{2}}\right)}{\left(1-\frac{(\gamma-1) \omega_{p e}^{2}+\omega_{u}^{2}}{\gamma^{2} \omega_{0}^{2}}\right)}+\frac{\omega_{c e}}{\gamma \omega_{0}}\right] E_{y} .
$$

and

$$
v_{0 y}=\frac{-i e}{m_{0} \gamma \omega_{0}\left(1-\frac{\omega_{c e}^{2}}{\gamma^{2} \omega_{0}^{2}}\right)}\left[1+\frac{\left(\frac{\omega_{c e}^{2}}{\gamma^{2} \omega_{0}^{2}}\right)\left(\frac{\omega_{p e}^{2}}{\gamma \omega_{0}^{2}}\right)}{\left(1-\frac{(\gamma-1) \omega_{p e}^{2}+\omega_{u}^{2}}{\gamma^{2} \omega_{0}^{2}}\right)}\right] E_{y} .
$$

Taking the complex conjugates $v_{0 x}^{*}$ and $v_{0 y}^{*}$ of $v_{0 x}$ and $v_{0 y}$ then substituting in Eq. (5) and Eq.(4), the relativistic factor $\gamma$ can be written as

$$
\gamma=1+\frac{1}{4}\left(\frac{e}{m_{0} \omega_{0} c}\right)^{2} \frac{1}{\left(1-\left(\omega_{c} / \omega_{0}\right)^{2}\right)}\left[\left(1-\frac{\omega_{c}^{2} \omega_{p}^{2}}{\omega_{0}^{2}\left(\omega_{0}^{2}-\omega_{u}^{2}\right)}\right)^{2}+\left(\frac{\omega_{c}}{\omega_{0}}\right)^{2}\left(1-\frac{\omega_{p}^{2}}{\left(\omega_{0}^{2}-\omega_{u}^{2}\right)}\right)^{2}\right] E_{y} E_{y}^{*} .
$$

The relativistic factor $\gamma$ may be written as following [38]

$\gamma=1+\alpha E_{y} E_{y}^{*}$.

By equating Eq.(15) and Eq.(16), we get

$\alpha E_{y} E_{y}^{*}=\frac{1}{4}\left(\frac{e}{m_{0} \omega_{0} c}\right)^{2} \frac{1}{4}\left(\frac{e}{m_{0} \omega_{0} c}\right)^{2} \frac{1}{\left(1-\left(\omega_{c} / \omega_{0}\right)^{2}\right)}\left[\left(1-\frac{\omega_{c}^{2} \omega_{p}^{2}}{\omega_{0}^{2}\left(\omega_{0}^{2}-\omega_{u}^{2}\right)}\right)^{2}+\left(\frac{\omega_{c}}{\omega_{0}}\right)^{2}\left(1-\frac{\omega_{p}^{2}}{\left(\omega_{0}^{2}-\omega_{u}^{2}\right)}\right)^{2}\right] E_{y} E_{y}^{*},(17)$

where, $\alpha$ refers to the relativistic nonlinearity which is occurring due to electron mass increment which oscillating in high intense laser field.

By putting the value of $\gamma$ from Eq. (16) into Eq. (4), we get

$$
\varepsilon_{r}=1-\frac{\frac{\omega_{p e}^{2}}{\omega_{0}^{2}}\left(1-\frac{\omega_{p e}^{2}}{\omega_{0}^{2}}\right)}{\left(1-\frac{\omega_{u}^{2}}{\omega_{0}^{2}}\right)}+\left[\frac{\frac{\omega_{p e}^{2}}{\omega_{0}^{2}}\left(\left(1-\frac{\omega_{p e}^{2}}{\omega_{0}^{2}}\right)^{2}+\frac{\omega_{c e}^{2}}{\omega_{0}^{2}}\right)}{\left(1-\frac{\omega_{u}^{2}}{\omega_{0}^{2}}\right)^{2}}\right] \alpha E_{y} E_{y}^{*} .
$$

In the above equation $\varepsilon_{r}$ includes the non relativistic part $\varepsilon_{0}$ and the relativistic part $\varepsilon_{2}$, 
where,

$$
\begin{gathered}
\varepsilon_{0}=1-\frac{\frac{\omega_{p e}^{2}}{\omega_{0}^{2}}\left(1-\frac{\omega_{p e}^{2}}{\omega_{0}^{2}}\right)}{\left(1-\frac{\omega_{u}^{2}}{\omega_{0}^{2}}\right)}, \\
\varepsilon_{2}=\left[\frac{\frac{\omega_{p e}^{2}}{\omega_{0}^{2}}\left(\left(1-\frac{\omega_{p e}^{2}}{\omega_{0}^{2}}\right)^{2}+\frac{\omega_{c e}^{2}}{\omega_{0}^{2}}\right)}{\left(1-\frac{\omega_{u}^{2}}{\omega_{0}^{2}}\right)^{2}}\right] \alpha .
\end{gathered}
$$

The wave equation of laser beam inside the plasma can be written as follows:

$$
\nabla^{2} \vec{E}_{0}=\nabla\left(\nabla \cdot \vec{E}_{0}\right)-\frac{\omega_{0}^{2}}{c^{2}}\left(\varepsilon_{r} \cdot \vec{E}_{0}\right)
$$

Using Eq.(9) and Eq.(10) we can write[36]

$\nabla \cdot \vec{E}_{0}=\frac{-\varepsilon_{x y}}{\varepsilon_{x x}}\left(\frac{\varepsilon_{x y}}{\varepsilon_{x x}} \frac{\partial E_{y}}{\partial y}+\frac{\partial E_{y}}{\partial x}\right)$.

Substituting Eq. (18) and Eq. (22) into Eq. (21)

$$
\frac{\partial^{2} E_{y}}{\partial x^{2}}+\frac{\partial^{2} E_{y}}{\partial z^{2}}=\left(k_{0}^{2}+\frac{\omega_{0}^{2}}{c^{2}} \varepsilon_{2} E_{y} E_{y}^{*}\right) E_{y} \text {, }
$$

where the products of nonlinear terms with second order space derivatives have been ignored and assuming $\frac{\partial}{\partial y}=0$ in two dimensional beam case.

To solve Eq.(23) we introduce

$$
E_{y}=\mathrm{A}_{\mathrm{y}} \exp -i\left(\omega_{0} t-k_{0} x\right) \text {, }
$$

where $A_{y}$ is a function of space which may be written as [7]

$$
\mathrm{A}_{\mathrm{y}}=\mathrm{A}_{0}(x, z) \exp i\left(k_{0} S\right) \text {, }
$$

$\mathrm{A}_{0}$ is a real function and $S$ is the phase of the laser beam.

Putting Eq.(24) and Eq.(25) in Eq.(23) and on solving for the real and imaginary parts we get

$$
\begin{gathered}
2 \frac{\partial S}{\partial x}+\left(\frac{\partial S}{\partial z}\right)^{2}=\frac{\varepsilon_{2}}{\varepsilon_{0}} A_{0}^{2}+\frac{1}{k_{0}^{2} A_{0}} \frac{\partial^{2} A_{0}}{\partial z^{2}}, \\
\frac{\partial A_{0}^{2}}{\partial x}+\left(\frac{\partial S}{\partial z}\right)\left(\frac{\partial A_{0}^{2}}{\partial z}\right)+\frac{\partial^{2} S}{\partial z^{2}} A_{0}^{2}=0,
\end{gathered}
$$

Assuming $S=\frac{1}{2} z^{2} \beta(x)+\phi$ and introducing Gaussian laser beam $A_{0}^{2}=\frac{E_{00}^{2}}{f_{0}} \exp \left(\frac{-z^{2}}{r_{0}^{2} f_{0}}\right)$

where $\beta(x)$ is the curvature of the wave front and $f_{0}(x)$ beam width parameter also using paraxial ray approximation then substituting in Eq.(27) we get $\beta(x)=\frac{1}{f_{0}} \frac{d f_{0}}{d x}$. 
Employing $\quad \mathrm{A}_{0}^{2}$ and $\beta(\mathrm{x})$ in Eq. (26) thus for initially plane wavefront conditions (i.e. $f_{0}=1$ and $\frac{d f_{0}}{d x}=0$ at $x=0$ ) we obtain

$$
\frac{d^{2} f_{0}}{d x^{2}}=\frac{1}{R_{d}^{2} f_{0}^{3}}-\frac{\varepsilon_{2} E_{00}^{2}}{\varepsilon_{0} r_{0}^{2} f_{0}^{2}},
$$

where $R_{d}=k_{0} r_{0}^{2}$ represents diffraction length and $r_{0}$ is the initial radius beam.

The first term in RHS of Eq. (28) is responsible for the diffraction of the beam (linear term) and the other term is the converging term (nonlinear term) resulting in self focusing of the laser beam. When the initial laser power is greater than critical power, the nonlinear term will overcome the linear term and hence the laser beam spot size $f_{0}$ will decrease along beam propagation direction. One can expect that at the balance of the diffraction and converging terms the laser beam will propagate inside plasma with constant spot size.

\section{THZ Dynamics}

The mechanism of the THz wave $\left(\overrightarrow{\mathrm{E}}_{\mathrm{t}}, \omega_{t}, \vec{k}_{t}\right)$ production depends upon the nonlinear interaction between x-mode high intense laser beam $\left(\overrightarrow{\mathrm{E}}_{0}, \omega_{0}, \vec{k}_{0}\right)$ and the UHW $\left(\overrightarrow{\mathrm{E}}_{1}, \omega_{1}, \vec{k}_{1}\right)$ introducing the phase matching, $\vec{k}_{t}=\vec{k}_{0}-\vec{k}_{1}$ and $\omega_{t}=\omega_{0}-\omega_{1}$ conditions. The electric fields for the UHW and THz waves are given by-

$$
\vec{E}_{1}=\hat{x} E_{1} \exp -i\left(\omega_{1} t-k_{1} x\right)
$$

$\vec{E}_{t}=\hat{y} E_{t} \exp -i\left(\omega_{t} t-k_{t} x\right)$,

where $\left(k_{1}=\sqrt{\left(\omega_{0}^{2}-\omega_{u}^{2}\right) / \mathrm{v}_{\mathrm{th}}^{2}}\right)$ is the propagation wave vector of UHW and $\left(v_{t h}=\sqrt{k_{B} T_{e} / m_{0}}\right)$ is electron thermal velocity.

The essential equations which govern the interactions between above electric fields inside plasma are

(a) The momentum equation

$\left.m_{j}\left[\frac{\partial \vec{v}_{j}}{\partial t}+{ }_{j} \cdot \vec{\nabla}\right) \vec{v}_{j}\right]=e_{j}\left[\vec{E}+\frac{1}{c} \vec{v}_{j} \times\left(\vec{B}_{0}+\vec{B}\right)\right]-\frac{k_{B} T_{j} \vec{\nabla} n_{j}}{m_{j} n_{0}}$,

(b) The continuity equation

$\frac{\partial n_{j}}{\partial t}+\vec{\nabla} \cdot\left(n_{j} \vec{v}_{j}\right)=0$

(c) Faraday equation

$\vec{\nabla} \times \vec{E}=-\frac{1}{c} \frac{\partial \vec{B}}{\partial t}$

(d) Ampere equation

$\vec{\nabla} \times \vec{B}=\frac{4 \pi}{c} \vec{J}+\frac{1}{c} \frac{\partial \vec{E}}{\partial t}$,

where $m_{j}, v_{j}, T_{j}, n_{j}$ denotes the mass, fluid velocity, temperature and the particle density of $j^{\text {th }}$ species and $j$ stand for electron and ions respectively. $k_{B}$ and $c$ are Boltzmann constant and light velocity in vacuum. $\vec{B}_{0}$ is the external magnetic field whereas $\vec{E}$ and $\vec{B}$ are the electric and magnetic fields of waves.

The total current density $\mathbf{J}_{\mathrm{t}}$ for $\mathrm{THz}$ field $\mathrm{E}_{\mathrm{t}}$ may be governed by the following wave equation [39]

$\frac{\partial^{2} E_{t}}{\partial x^{2}}=\frac{4 \pi}{c^{2}} \frac{\partial J_{t}}{\partial t}+\frac{1}{c^{2}} \frac{\partial^{2} E_{t}}{\partial t^{2}}$ 
the total current density $\vec{J}_{t}$ is given by

$\vec{J}_{t}=e n_{0}\left(\vec{v}_{t y}^{i}-\vec{v}_{t y}^{e}\right)-\left(\frac{1}{2}\right) e\left(\frac{n_{u}^{*}}{n_{0}}\right) n_{0} \mathrm{v}_{\mathrm{y}}$,

where $n_{u}$ represent fluctuation density in UHW, superscript * denotes the complex conjugate of that quantity. In low frequency field, using Eq.(31), the ion and electron velocities $\vec{v}_{t y}^{i}$ and $\vec{v}_{t y}^{e}$ may be given as

$\vec{v}_{t y}^{i}=\left(1-\frac{\omega_{c i}^{2}}{\gamma^{2} \omega_{t}^{2}}\right)^{-1}\left(\frac{i e}{\omega_{t} \gamma m_{i}} \vec{E}_{t y}+\frac{\omega_{c i}}{\gamma^{2} \omega_{t}^{2}} \frac{k_{B} T_{i}}{m_{i}} \nabla_{x}\left(\frac{n_{t}}{n_{0}}\right)\right)$,

$\vec{v}_{t y}^{e}=\left(1-\frac{\omega_{c e}^{2}}{\gamma^{2} \omega_{t}^{2}}\right)^{-1}\left(\frac{-i e}{\omega_{t} \gamma m_{0}} \vec{E}_{t y}+\frac{\omega_{c e}}{\gamma^{2} \omega_{t}^{2}} \frac{k_{B} T_{e}}{m_{0}} \nabla_{x}\left(\frac{n_{t}}{n_{0}}\right)\right)$,

where $n_{t}$ is the fluctuation density in THz field.

Using continuity equation, the fluctuation density $n_{u}$ may be written as:

$n_{u}=n_{0} \frac{k_{1}}{\omega_{1}} v_{1}$

here, $\vec{v}_{1}=\frac{i e \vec{E}_{1} \omega_{1}}{m_{0}\left(\omega_{1}^{2}-\omega_{c e}^{2}\right)}$ is the electron velocity in UHW field.

Substituting equation of fluctuation density $n_{u}$ and Eqs. (37) and (38) in Eq.(36), one can rewrite Eq. (35) to be

$\left(\frac{\partial^{2} \mathrm{E}_{\mathrm{ty}}}{\partial x^{2}}-\frac{1}{c^{2}} \frac{\partial^{2} \mathrm{E}_{\mathrm{ty}}}{\partial t^{2}}-\frac{1}{\mathrm{~V}_{\mathrm{A}}^{2}} \frac{\partial^{2} \mathrm{E}_{\mathrm{ty}}}{\partial t^{2}}\right)=-\frac{4 \pi n_{0} T_{e}}{B_{0}^{2}} \frac{\partial^{2} \mathrm{E}_{\mathrm{ty}}}{\partial x^{2}}+i \omega_{t} \frac{4 \pi n_{0} e}{c^{2}} \frac{1}{2} \mu^{*} \mathrm{v}_{\mathrm{x}}$,

where $\mu=\frac{n_{u}}{n_{0}}$ is normalized fluctuation density of UHW and $V_{A}=\sqrt{B_{0}^{2} /\left(4 \pi n_{0} \gamma m_{i}\right)}$ is Alfvén velocity.

It is important to mention that Eq.(39) has been written by introducing a quasi-neutrality condition $\left(n_{i}=n_{e}=n_{0}\right)$ and cold ion plasma $\left(T_{i}=0\right)$ also by assuming $\left(\omega_{t}^{2} \square k_{t}^{2} v_{t h}^{2}\right)$ and $\left(\omega_{t}^{2} \square \omega_{c e}^{2}\right) \cdot n_{0}$ is background density of plasma.

We can get Eq. (39) in the following form

$\frac{\partial^{2} E_{t y}}{\partial x^{2}}\left[\frac{\omega_{t}^{2}}{c^{2}}-\frac{\omega_{p e}^{2} k_{t}^{2}}{c^{2} \omega_{c e}^{2}} \frac{k_{B} T_{e}}{m_{0}}+\frac{\omega_{t}^{2}}{V_{A}^{2}}\right] E_{t y}=\frac{e}{2 m_{e} c^{2}\left(\omega_{1} \omega_{0}\right)^{3 / 2}} \frac{1}{f_{0}}\left(1+\left(\omega_{c e}^{2} \omega_{p e}^{2} /\left(\omega_{0}^{2}-\omega_{u}^{2}\right)\right)\right) \omega_{p e}^{2}\left(\omega_{0}-\omega_{1}\right) k_{1} E_{y}^{2}$.

\section{Discussion of Numerical Results}

We have solved equation Eq. (28) numerically by using initial conditions $f_{0}=1$ and $\frac{d f_{0}}{d x}=0$ at $x=0$, by using the following set of parameters from experimental observations [40]:Pump beam intensity $(I)=10^{18} \mathrm{~W} / \mathrm{cm}^{2}, r_{0}=10 \mu \mathrm{m}, \omega_{0}=2.395 \times 10^{15} \mathrm{rad} / \mathrm{sec}, c / \omega_{p}=0.1285 \mu \mathrm{m}$

$\omega_{1}=2.393 \times 10^{15} \mathrm{rad} / \mathrm{sec}$. In Eq. (28), the diffraction phenomenon leads to the divergence of the beam and second term arises due to the relativistic non linearity which is responsible for self-focusing. The difference in magnitude of these two terms, governs the focusing/defocusing behaviour of the beam. If the magnitude of the first term exceeds the second term, then divergence of the beam occurs and for a reverse situation focusing of the beam is observed. 
Figure (1) is the plot between beam width parameter $f_{0}$ and $\xi$ for three distinct values of magnetic field viz. $\mathrm{B}_{0}=4 \cdot 6,4.7$ and $4.8 \mathrm{MG}$. The diffraction of beam begins with increasing the magnetic field. This is due to dominance of diffraction term over the non-linear self-focusing term with increase in magnetic field. Fig. 1 shows that the self-focusing length reduces with magnetic field because quasi stationary magnetic field is generated at relativistic power. There is an increase in the self focusing with plasma density because at very high power, relativistic electrons propagate with the laser pulse, which produces a huge amount of current, resulting an enhancement in the self focusing due to the higher pinching effect of magnetic field.

Now our goal here is to make theoretical predictions that are possible to verify experimentally for efficient methods for qualitative as well as quantitative calculations for $\mathrm{THz}$ generation. For this purpose, we solve Eq. (40), numerically by using the above parameters.

Figure 2 depicts the $\mathrm{THz}$ field variation versus the normalized distance of propagation when laser beam exhibit oscillatory focusing at varying magnetic field. There is an enhancement in the normalized $\mathrm{THz}$ amplitude by increasing the magnetic field. These parameters, for laser frequency $\omega_{0}=2.395 \times 10^{15} \mathrm{rad} / \mathrm{sec}, \quad$ plasma frequency $c / \omega_{p}=0.1277 \mu \mathrm{m}, \quad$ correspond to $\mathrm{B}_{0}=4.6,4.7$ and 4.8 MG. The huge magnetic fields of up to $10^{9} \mathrm{G}$ [41-45] have been observed in the regards of $\mathrm{THz}$ generation [46] in nonlinear coupling of high -intensity short-pulse laser and plasmas. We find that the $\mathrm{THz}$ wave has the fields approximately $0.04194 E_{00}, 0.08316 E_{00}$ and $0.2827 E_{00}$ for the different values of magnetic field $\mathrm{B}_{0}=4.6,4.7$ and $4.8 \mathrm{MG}$ as shown in Fig. 2. The generated field at $\mathrm{THz}$ frequency in the current scheme is larger than some other schemes of $\mathrm{THz}$ generation $[47,48]$.

THz radiation power spectres' are shown in Fig. (3) for increasing values of magnetic field $\mathrm{B}_{0}=4.8,4.9$ and $5 \mathrm{MG}$. In this figure $\mathrm{THz}$ field acquires a larger value as electron cyclotron frequency increases. The THz power comes out to be $1.262 \times 10^{-4} P_{0}, 1.884 \times 10^{-3} P_{0}, 3.011 \times 10^{-4} P_{0}$, for the different values of magnetic field, where $P_{0}$ is the incident laser power.

\section{Conclusion}

A significant effect of relativistic self-focusing of $\mathrm{x}$-mode laser beam (with paraxial approximation) on the amplitude and power of $\mathrm{THz}$ generation has been observed. We have also shown that the increase in the laser intensity due to self-focusing effect leads to higher amplitude of $\mathrm{THz}$ radiation and hence $\mathrm{THz}$ generation is dominated by self-focusing of laser beam. The resulting self focused beam couples with the UHW which produces to generate the $\mathrm{THz}$ frequency radiation. The magnetic field also affects the nonlinear coupling. The self focusing of a x-mode relativistic laser beam in magneto plasma is significantly enhanced by the ambient magnetic field. We get the enhancement in the $\mathrm{THz}$ wave amplitude with increase in the magnetic field because of the linear dependency of $\mathrm{THz}$ radiation amplitude on the $\mathrm{x}$-mode laser amplitude obvious from Eq. (40).

\section{Acknowledgment}

This work was partially supported by University Grant Commission (UGC), India, and Ministry of Higher Education and Scientific Research, Government of Iraq.

[1] B Ferguson and X. C. Zhang, Nat. Mater., 1, 26 (2002).

\section{References}

[2] Y. C. Shen, T.W. P. F. Today, B. E. Cole, W. R. Tribe and M. C., Kemp, Appl. Phys. Lett. 86, 241116 (2005).

[3] H. Zheng, A. Redo-Sanchez, and X. C. Zhang, Opt. Express 14, 9130 (2006).

[4] W. P. Leemans, J. van Tilborg, J. Faure, C. G. R. Geddes, C Toth., C. B. Schroeder, E. Esarey and G. Fubiani, Phys. Plasmas, 11, 2899 (2004).

[5] J. T. Mendonca, New Journal of Physics, 8, 185 (2006)

[6] E. Budiarto, J. Margolies, S. Jeong, J. Son, and J. Bokor, IEEE J. Quantum Electron. 32, 1839(1996).

[7] S A Akhmanov, AP Sukhorov, RV Khokhlov. Sov Phys Usp, 10, 609-36 (1968).

[8] M S Sodha, A K Ghatak, V K Tripathi. Prog Opt, 13. New-York: Academic Press; (1976). (p. 169-265).

[9] P Sprangle , E. Esarey, Phys Rev Lett, 67, 2021-4 (1991)

[10] H M Milchberg, III CG Durfee, T J Mcllrath, Phys Rev Lett, 75, 2494-7 (1995).

[11] P Mulser, D. Bauer, Laser Part Beams, 12 , 22-5 (2004).

[12] H. Hora, Laser Part Beams, 25, 37-45(2007)

[13] AV Gurevich, Berlin, Springer (1978).

[14] F W Perkins, M V Goldman, J Geophys Res, 86,600-8 (1981)

[15] H Y Niu, X T He , B Qiao, CT Zhou., Laser Part Beams, 26, 51-9 (2008)

[16] J X Lee, W P Zang, Y D Li, J G Tian. Opt Exp, 17, 11850-9 (2009).

[17] H. D. Ladouceur, A. P. Baronavski, D. Lohrmann, P. W. Grounds, and P. G. Girardi, Opt. Commun., 187,107 (2001). 
[18] C. D. Amico, A. Houard, M. Franco, B. Prade, A. Mysyrowicz, A. Couairon, and V. T. Tikhonchuk, Phys. Rev. Lett. 98, 235002 (2007).

[19] S. Tzortzakis, G. Me'chain, G. Patalano, Y.-B. Andre', B. Prade, M. Franco, A. Mysyrowicz, J.-M. Munier, M. Gheudin, G. Beaudin, and P. Encrenaz, Opt. Lett. 27, 1944 (2002).

[20] A. Houard, Y. Liu, B. Prade, V. T. Tikhonchuk, and A. Mysyrowicz, Phys. Rev. Lett. 100, 25500(2008).

[21] P. Sprangle, J. R. Penano, B. Hafizi, and C. A. Kapetanakos, Phys. Rev. E, 69, 066415 (2004).

[22] Y. Liu, A. Houard, B. Prade, S. Akturk and A. Mysyrowicz, Phys. Rev. Lett., 99, 135002 (2007); Y. Liu , A. Houard , B. Prade, A. Mysyrowicz, A. Diaw and V. T. Tikhonchuk Appl. Phys. Lett., 93, 051108 (2008).

[23] Wang, J.-F. Daigle, S. Yuan, F. The'berge, M. Cha^teauneuf, J. Dubois, G. Roy, H. Zeng, and S. L. Chin,Phys. Rev. A, 83, 053801 (2011).

[24] H. C. Wu, J. Meyer-ter-Vehn, H. Ruhl, and Z. M. Sheng, Phys. Rev. E, 83, 036407 (2011).

[25] J. L. Hughes, by H. Schwarz, H. Hora (Plenum, New York, 1974), p. 849.

[26] H. Hora, , Opto-Electronics 5, 491 (1973).

[27] H. Hora, J. Opt. Soc. Am. 65, $882(1975)$.

[28] K.A. Tanaka, R. Kodama, H. Fujita, M. Heya, N. Izumi, Y. Kato, Phys. Plasmas 7, 2014 (2000).

[29] J. Fuchs, J.C. Adam, F. Amiranoff, S.D. Baton, N. Blanchot, P. Gallant, L. Gremillet, A. Héron, J.C. Kieffer, G. Laval, G. Malka, J.L. Miquel, P. Mora, H. Pépin, C. Rousseaux, Phys. Plasmas 6, 2563 (1999).

[30] P. Monot, T. Auguste, P. Gibbon, F. Jakober, G. Mainfray, A. Dulieu, M. Louis- Jacquet, G. Malka, J.L. Miquel, Phys. Rev. Lett. 74, 2953 (1995).

[31] D.A. Jones, E.L. Kane, P. Lalousis, P. Widles, H. Hora, Phys. Fluids 25, 2295 (1982)

[32] T. Hauser, W. Scheid, H. Hora, Phys. Rev. A 45, 1278 (1992)

[33] P. Gibbon, E. Forster, Plasma Phys. Control. Fusion 38, 769 (1996).

[34] D. Umstadter, J. Phys. D 36, 151 (2003)

[35] A Pukhov, J. Meyer-ter-vehn, Phys Rev Lett, 76, 3975-8(1996).

[36] ] K. I. Hasson, A. K. Sharma and R. A Khamis, Phy.Scr., 81, 025505 (2010).

[37] A. K. Sharma, Appl. Phys., 49, 4 (1978).

[38] Hyder A. Salih, R. P. Sharma and M. Rafat, Physics of Plasma, 11, 6, (2004).

[39] [P.K. Shukla and R. P. Sharma ,Phys. Rev.A, 25, 2816-2819 (1982).

[40] S. Mondala, V. Narayanana, W. J. Dingb, A. D. Lada, B Haob, S Ahmada, W. M. Wangb, Z. M. Sheng, S. Senguptad, P. Kawd, A. Das, and G. R. Kumara, www.pnas.org/cgi/doi/10.1073/pnas.1200753109.

[41] [41] S. C. Wilks, W. L. Kruer, M. Tabak, and A. B. Langdon, Phys. Rev. Lett. 69, 1383 (1992).

[42] [42] R. N. Sudan, Phys. Rev. Lett. 70, 3075 (1993)

[43] [43] A. S. Sandhu, A. K. Dharmadhikari, P. P. Rajeev, G. R. Kumar, S. Sengupta, A. Das, and P. K. KaW, Phys Rev Lett 89:225002. (2003).

[44] R. Rajeev, T. Madhu Trivikram, K. P. M. Rishad, V. Narayanan, E. Krishnakumar and M. Krishnamurthy* Nature Physics, DOI: 10.1038/NPHYS2526.

[45] U. Wagner, M. Tatarakis, A. Gopal, F. N. Beg, E. L. Clark, A. E. Dangor, R. G. Evans, M. G. Haines, Physical Review E 70, 026401 (2004).

[46] H. Hamster, A. Sullivan, S. Gordon, W. White, and R. W. Falcone, Phys.Rev. Lett. 71, 2725 (1993); H. Hamster, A. Sullivan, S. Gordon, and R. W. Falcone, Phys. Rev. E 49, 671 (1994).

[47] T Bartel, P Gaal, K Reimann, M Woerner, and T Elsacsser, Opt. Lett., 30, 2805(2005)

[48] D. You, R. R. Jones, P. H. Bucksbaum, and D. R. Dykaar, Opt. Lett., 18, (1993) 290.

\section{FIGURE CAPTION}

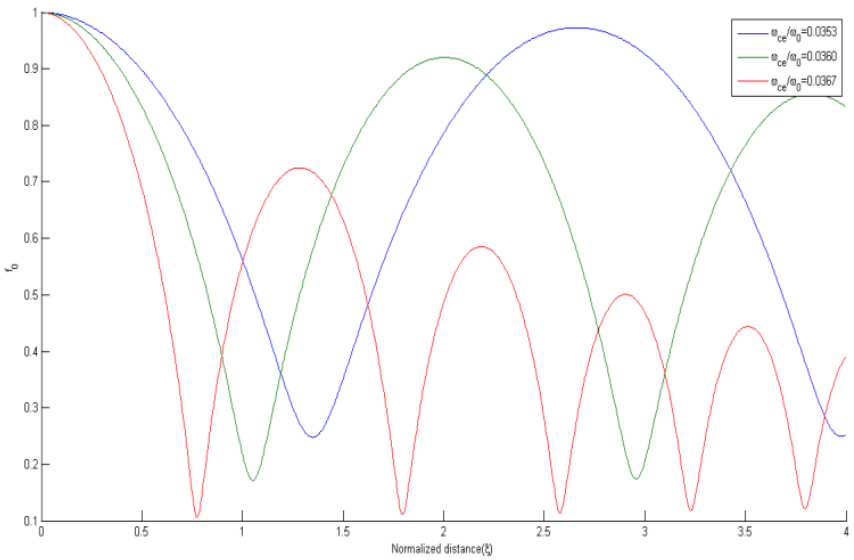

FIG. 1

FIG.1. (Color online) Variation of beam width parameter $f_{0}$ with normalized distance $\xi=\frac{x}{k_{0} r_{0}^{2}}$. when,

$\mathrm{E}_{00=} 5 \times 10^{9} \mathrm{~V} / \mathrm{cm}, \omega_{0}=2.3951 \times 10^{15} \mathrm{rad} / \mathrm{sec}, \omega_{p} / \omega_{0}=0.9812$ and $\alpha E_{00}^{2}=4$, for different values of electron cyclotron frequency $\omega_{c e} / \omega_{0}=0.0353,0.0360,0.0367$. 


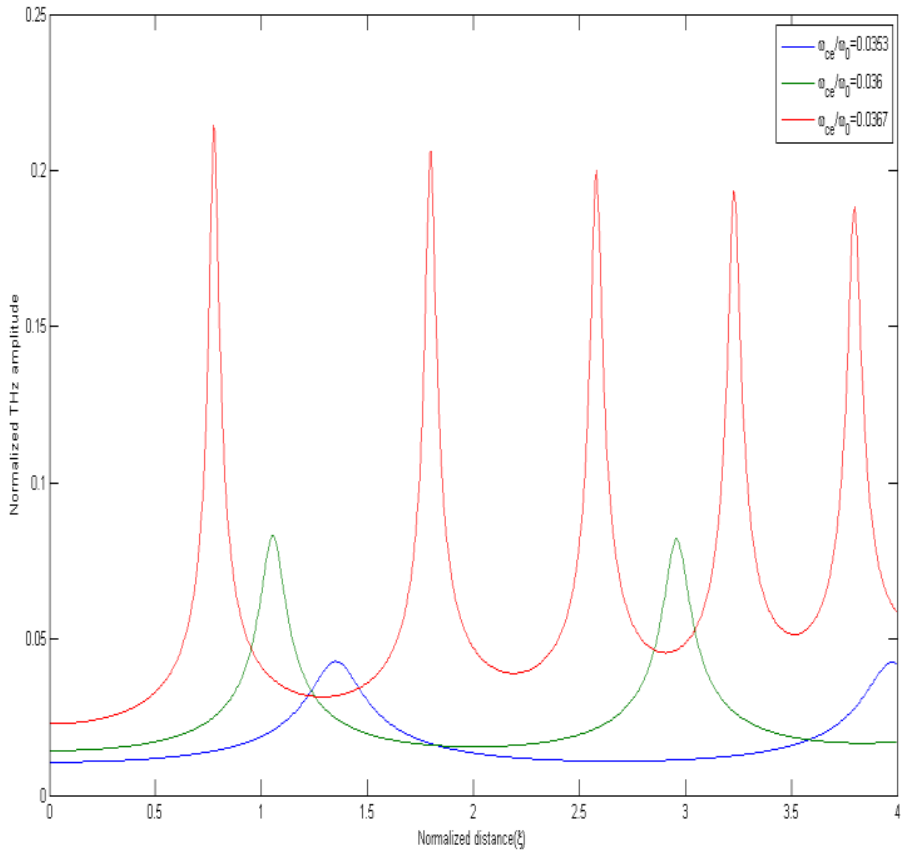

FIG. 2

FIG.2. Normalized THz radiation field amplitude and vs. normalized distance for different values of electron cyclotron frequency $\omega_{c e} / \omega_{0}=0.0353,0.0360,0.0367$ for the same parameters as in Fig. 1.

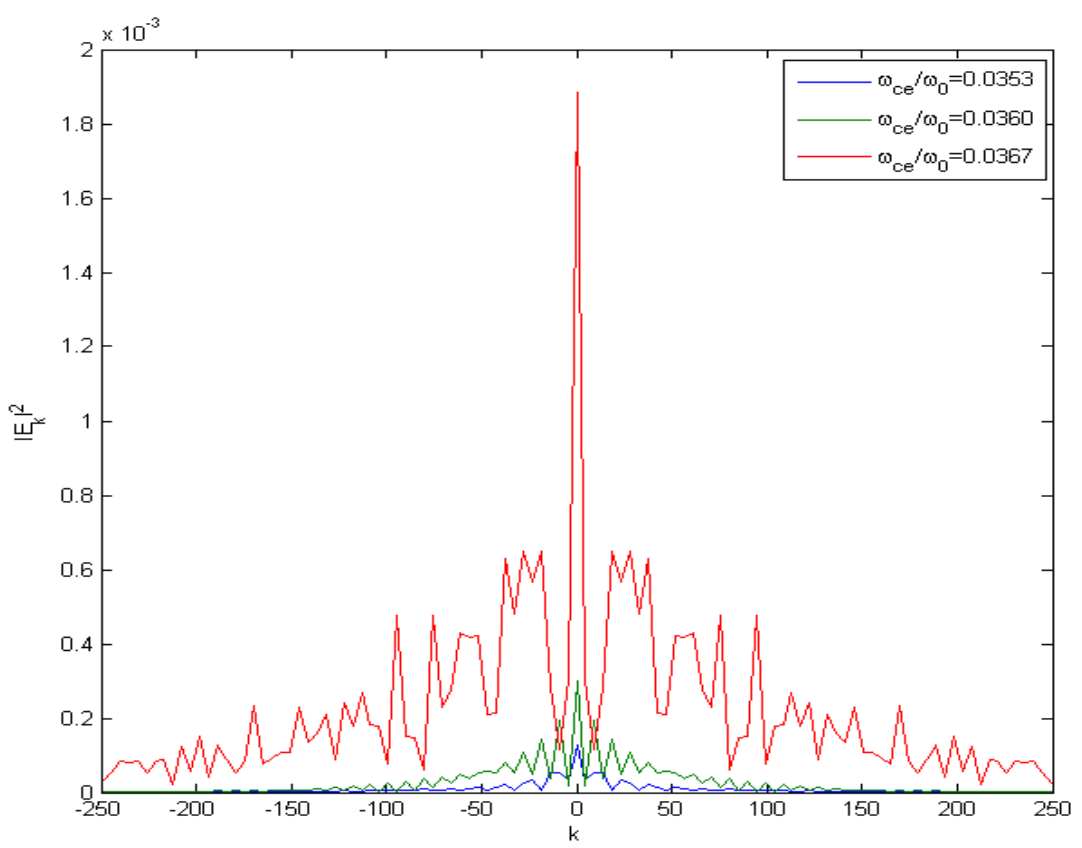

FIG. 3.

FIG.3. Power spectra of $\mathrm{THz}$ radiation at the end of simulation for different values of magnetic field $\mathrm{B}_{0}=4.8,4.9$ and $5 \mathrm{MG}$ for the same parameters as in Fig. 1. 